\title{
The possible connection between ionization in the atmosphere by cosmic rays and low level clouds
}

\author{
E. Palléa,*, C.J. Butler ${ }^{\mathrm{b}}$, K. O'Brien ${ }^{\mathrm{c}}$ \\ ${ }^{a}$ Big Bear Solar Observatory, New Jersey Institute of Technology, 40386 North Shore Lane, Big Bear City, CA 92314, USA \\ b Armagh Observatory, College Hill, BT61 9DG. Armagh, N. Ireland, UK \\ ${ }^{\mathrm{c}}$ Department of Physics and Astronomy, Northern Arizona University PMB 1019, 2675 W. Hwy. 89A, Sedona, AZ 86336-5240, USA
}

Received 29 September 2003; received in revised form 23 March 2004; accepted 4 June 2004

Available online 15 September 2004

\begin{abstract}
Recent analysis of monthly mean cloud data from the International Satellite Cloud Climatology Project uncovered a strong correlation between low cloud and the cosmic ray flux for extensive regions of the Earth. Additional data have been recently released covering the period up to September 2001 with which we have made a new study of the geographical variation of the correlation between low cloud and predicted ionization level from cosmic rays at an altitude of $2 \mathrm{~km}$. When analysed globally, we find that the correlations do not correspond to the latitude variation of cosmic ray flux and they are not field significant. Nonetheless they appear to be marginally field significant over broad latitude and longitude bands with a peak positive correlation at 50 degrees North and South and a tendency to negative correlation at lower latitudes. The correlation is strongest over the North and South Atlantic. Several of these features are consistent with the predictions of the electroscavenging process.

We use a simple model to calculate the climatic impact should the correlation be confirmed. We show that, under the most favorable conditions, a reduction in low cloud cover since the late 19th century, combined with the direct forcing by solar irradiance can explain a significant part of the global warming over the past century, but not all. However, this computation assumes that there is no feedback or changes in cloud at other levels.
\end{abstract}

(C) 2004 Elsevier Ltd. All rights reserved.

Keywords: Clouds; Cosmic rays; Climate; Solar activity; Global warming

\section{Introduction}

Galactic cosmic rays (GCR) are known to be the principal agents of ionization in the atmosphere above $1 \mathrm{~km}$ altitude. This has led to the suggestion that cloud formation may be affected by cosmic rays through an enhanced production of charged aerosols that may grow to become cloud condensation nuclei. As the cosmic ray flux on Earth is strongly modulated by solar activity, in

\footnotetext{
*Corresponding author.

E-mail addresses: epb@bbso.njit.edu (E. Pallé), cjb@star. arm.ac.uk (C.J. Butler), Keran.O'Brien@nau.edu (K. O'Brien).
}

the sense that increased solar activity leads to a reduction in the cosmic ray flux, if cosmic rays do affect cloudiness, they could provide a link through which solar activity affects climate.

The study by Svensmark and Friis-Christensen (1997) suggested that total cloud cover over the mid-latitude oceans was strongly correlated with the cosmic ray flux measured by neutron monitors - at least over the period 1984-1991 covered by the satellite cloud data they studied. However, the method by which they had fitted together different satellite cloud datasets, led to some criticism of their conclusions (Kernthaler et al., 1999). 
Subsequently, a new uniformly calibrated series of satellite cloud observations from the International Satellite Cloud Climatology Project (ISCCP), the ISCCP-D2 series, has made it possible to assess the variability of cloud cover over substantial parts of the world and to compare this with the observed cosmic ray flux for a longer time interval. The good correlation between total cloud cover and cosmic ray flux found by Svensmark and Friis-Christensen (1997) for the period 1984-1991 was confirmed by this data, however, after 1991 the correlation disappeared.

From this data, only the low cloud of altitude $1-3 \mathrm{~km}$ correlated with cosmic ray flux; mid-level and higher cloud showed no apparent correlation (Pallé and Butler, 2000; Marsh and Svensmark, 2000). It was further shown that, although the degree of variation was small ( $\sim 1 \%$ global cloud coverage), if it persisted over decadal time scales, it could lead to significant changes in the global energy budget. Indeed, if the correlation observed between cosmic ray flux and low cloud cover during the 11 years 1983-1994 had been operational since the late 19 th century and there were no feedback mechanisms operating or other cloud changes, then the observed variation in solar activity and cosmic ray flux could conceivably account for an appreciable fraction of global warming observed over the past century (Pallé and Butler, 2000).

Little is known about the physical mechanism that may link cosmic rays and clouds but, if one were to exist, it seems likely that it may occur, either through the effect of ionization on cloud formation or ion-mediated nucleation (IMN) which may result in the formation of condensation nuclei and increases in cloud lifetime ( $\mathrm{Yu}, 2002$; Turco et al., 1998; Svensmark and FriisChristensen, 1997) or through the influence of cosmic rays on the global electrical circuit, the electroscavenging effect (Tinsley et al., 2000). For a detailed discussion on both mechanisms see Tinsley and $\mathrm{Yu}$ (2003). GCR have been reported to affect mainly low clouds (Pallé and Butler, 2000; Marsh and Svensmark, 2000), however, some authors have suggested that there may be an anti-correlation of high clouds with GCR if el Niño and volcano events are removed ( $\mathrm{Yu}, 2002$; Marsden and Lingenfelter, 2003).

There are other mechanisms proposed in the literature by which solar activity could affect Earth's cloudiness. Haigh (1996) proposed that changes in solar ultraviolet irradiance over a solar cycle may affect weather patterns in the troposphere via changes in the ozone layer and the propagation of planetary waves from the troposphere to the stratosphere. Udelhofen and Cess (2001) found a statistically significant 11 -year signal in total cloudiness over the United States. Here the cloud cover variations were found to be in phase with solar irradiance rather than GCR. These results suggest a modulation of the atmospheric circulation following the action of solar ultraviolet radiation on ozone (Haigh, 1996; Shindell et al., 1999). As we will see, over the United States, ISCCP low cloud cover data does not show any significant correlation with GCR. More recently, Kristjansson et al. (2002) have used the ISCCP D2 (1983-1999) dataset to assess the correlation between low cloud cover with total solar irradiance (TSI) and GCR. They conclude that TSI correlates better and more consistently with low clouds than does GCR, and they provide a possible physical explanation following the results of Haigh (1996) and those of White et al. (1997) who found a significant solar signal in multi-decadal time series of sea surface temperature (SST). A review of the several mechanisms by which solar activity could influence cloud and climate has been published by Carslaw et al. (2002).

In this paper we concentrate on the hypothesis that GCR are responsible for the coupling of low cloudiness and solar activity through their effects on cloud processes. We have not explored the correlation with TSI. However, it is the belief of the authors that a simple correlation analysis is, in any case, inadequate to unambiguously decide which physical mechanism lies behind the correspondence.

Whereas in our earlier study (Pallé and Butler, 2000), using the ISCCP-D2 mean monthly data, we looked at the correlation of low cloud cover and cosmic ray flux over substantial areas of the globe (e.g., N. Atlantic, mid-high latitudes, global, etc.), in this study, with the data extended up to 2001, we are interested to see if the correlation is regional, if there are significant differences in sensitivity in different areas of the world and, in particular, if we can detect any variation with latitude that could be attributed to the differential shielding of cosmic ray particles by the Earth's magnetic field. If it were possible to quantify this, we hoped to be able to determine the coefficients of regression of the low cloud cover and cosmic ray flux over different regions to build up a more detailed picture of the contribution to climate change of cloud cover changes over the globe. Rather than use measured cosmic ray fluxes, which by and large are available only for relatively few points on the globe and usually over land regions only, we have used predicted fluxes from the LUIN Code. These have the added advantage that they can be extrapolated back to previous centuries using carbon and beryllium isotope proxies and used to predict ionization levels at different levels in the atmosphere.

\section{Data}

\subsection{Cloud data}

Continuous global datasets of mean fractional cloud coverage and cloud radiative properties have been provided by the ISCCP from the combined observations 
of several inter-calibrated geosynchronous and polar satellites. Data are collected from a suite of weather satellites operated by several nations and processed by several groups. All ISCCP data products are archived at the ISCCP Central Archive (isccp.giss.nasa. gov).

The first ISCCP-D dataset, which extended from July 1983 to August 1994, is a recalibration of the old ISCCP-C dataset, released in early 1999. An extension of this dataset up to September 2001 was released in 2002. Both datasets are available in daily $(\mathrm{C} 1, \mathrm{D} 1)$ and monthly (C2, D2) means. In the ISCCP dataset, total cloudiness is determined using both visible and infrared radiances, whereas the separation into low, mid and high level cloud types is determined using infrared radiances only. During day time, there is also a further distinction into 15 different cloud types (depending on cloud height and optical thickness) based on both visible and infrared radiances. The data are given for $280 \times$ $280 \mathrm{~km}^{2}$ cells with the cloud fraction in each cell determined by dividing the number of cloudy pixels by the total number of pixels per cell. Here, we use only the infra-red channel data available during the whole $24 \mathrm{~h}$ day. A detailed description of the ISCCP D dataset can be found in Rossow et al. (1996).

\subsection{Computed cosmic ray particle fluxes and ionization levels in the atmosphere}

The Linear Uniform Internuclear Transport (LUIN) 2000 Code computes the cosmic ray-produced particle fluxes and the level of ionization at different altitudes and geo-graphical location. LUIN incorporates an analytical theory of the transport of high-energy radiation through the earth's atmosphere. The theory is based on a solution of the Boltzmann transport equation separable into longitudinal and transverse components, applicable to high-energy hadron-nucleus collisions, and is based on work by Passow (1962) and reported by Alsmiller (1965), Elliott (1955) and Williams (1966). In this approach, all secondary particles other than hadrons are mediated by meson production and decay. As input, it uses maps of the Earth's magnetic field as a function of time and the Heliocentric Potential, an interplanetary magnetic field index first calculated by O'Brien (1979) which is based on the cosmic ray flux since 1938, the $a a$ index from 1868 to 1938 and prior to 1868 on the $\mathrm{C}_{14}$ isotope concentration in tree-rings. Monthly estimates of this index, for the period during which cloud data are available, are employed by the LUIN code to provide mean monthly values for the particle flux and ionization level at different heights in the atmosphere and in different geographical locations.

To reduce the amount of computer time required we have calculated the particle fluxes and ionization level for a grid of points at the centers of cells of size 15 degrees in latitude and 30 degrees in longitude, except for polar regions where the cells are 7.5 degrees in latitude and 60 degrees in longitude. The same cell boundaries have been used for the cloud data with which the cosmic ray fluxes and ionization levels are to be correlated. The particle fluxes and ionization levels have been computed at these cell centers at five different altitudes: $0 \mathrm{~km}$ (sea level), $2 \mathrm{~km}$ (mean height of low clouds), $4.5 \mathrm{~km}$ (mean height of mid-level clouds), $10.5 \mathrm{~km}$ (mean height of high clouds) and $16 \mathrm{~km}$.

The final output is a matrix of data over the Earth's surface with time. Monthly tables of the particle fluxes and ionization levels at the center points of our grid cells and at five different altitudes have been computed for the period 1957-1999. Previous to this; yearly means have been computed back to 1868 , and approximately 10 -year resolution data back to $1008 \mathrm{AD}$. As this data may be useful for other studies, we have made it available from http://climate.arm.ac.uk. Though we have used the LUIN code to calculate the fluxes of a variety of elementary particles, we are only concerned here with the calculated ionization levels at $2 \mathrm{~km}$ altitude, the mean height of low cloud.

As an indication of the quality of the agreement between the computed values using the LUIN code and observations, calculations for Palestine, Texas in 1969 and 1970 are compared with measurements by Lowder et al. (1971) in Fig. 1. A comparison with measurements over Durham, New Hampshire in 1969 is also shown in Fig. 1 (Lowder et al., 1971). All three sets of calculations used the 1965 geomagnetic epoch and the heliocentric potential appropriate for the time of the measurements. Agreement between the observed and predicted ionization as a function of height is quite good.

\section{Statistical methods}

In determining the correlation between low clouds and GCR a problem arises. Cloud data have a strong seasonal cycle which is not present in the flux of GCR. To overcome this problem, some previous studies (Pallé and Butler, 2000; Marsh and Svensmark, 2000) have used a 12-month running mean of the cloud data, however this enhances the serial correlation between data points and reduces the number of degrees of freedom. For smoothed data the equivalent number of degrees of freedom is difficult to estimate and, in this case, could be as low as 5 or 6 (Udelhofen and Cess, 2001).

A second approach is to use annual means. This is the method we have adopted in this paper. The annual mean low cloud cover was determined for the seventeen years July 1983-June 2000 by averaging the data for the twelve months July-June. These annual data points were then correlated with the mean computed particle fluxes 
Ionization Profile over Palestine, Texas

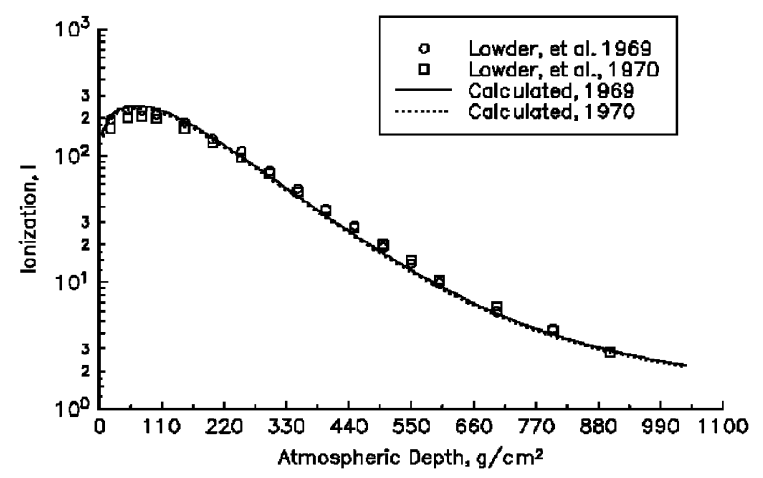

Ionization Profile over Durham, New Hampshire

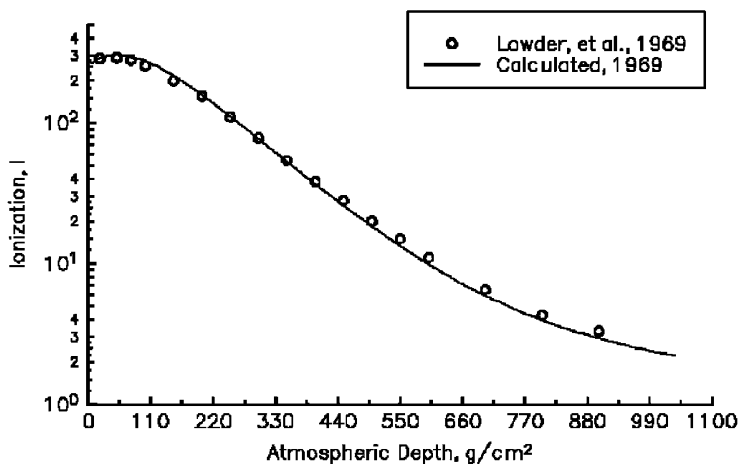

Fig. 1. Calculated and measured ionization by cosmic rays over Palestine, Texas in 1969 and 1970 (top) and Durham, New Hampshire in 1969 (bottom); adapted from Lowder et al. (1971). The ionization, or ion-pair production rate, is given as I, (ion-pairs $\mathrm{cm}^{-3} \mathrm{~s}^{-1}$ ) of air at standard temperature and pressure.

and ionization levels over the same period. Since the estimate of the significance of a given correlation depends critically on the number of degrees of freedom, a parameter difficult to estimate, we have calculated the significance of our correlations using Monte Carlo techniques. The cloud data for each of the grid cells is randomly ordered in time in 10,000 different permutations and the correlation coefficient between cloud and the ionization data for the same grid cell computed. The number of correlation coefficients of the randomized data higher than that obtained with the original data gives us an estimate of the significance of the result.

Cloud data from adjacent cells can also be correlated due to field auto-correlations in the dataset. Here again, we have used Monte Carlo techniques to determine the extent of field correlations. Annual data for each of the 144 cloud grid cells have been correlated with all other cells. We find, as might have been expected, that there is a field correlation in the cloud data. On average, for a given cloud cell, about $3-4 \%$ of all other cloud cells correlate with it with a significance level larger than $99 \%$ (factor 3) and about $12-14 \%$ correlate with a significance level larger than $95.0 \%$ (factor 3 again). Field correlations of this order would be expected due to similarity in the behavior of cloud coverage in geographically adjacent cells.

\section{The correlation between low clouds and ionization level in the atmosphere, 1983-2001}

Fig. 2 shows the global annual averages of GCRinduced ionization in the atmosphere and low cloud amounts for the period July 1983-June 2000 (ionization data is only updated to December 2000). A quick look at the data reveals the good agreement between those two quantities from 1983 to 1994, however, from 1995 to 2000 the correspondence breaks. The correlation coefficient (0.49) over the full period is significant only at the $\sim 85 \%$ level. There are several possible causes for the break of correlation after 1994, not least that a physical relationship between ionization and low cloud formation does not exist. However, it is worth mentioning that the new release of ISCCP data covers precisely the period 1995 onward, and increasing the mean level of the new data by only $+1 \%$ would return the correlation coefficient to 0.89 (99.9\% significance level). Some authors have suggested that the new (post-1994) ISCCP data may have a calibration error (Marsh and Svensmark, 2003), however, no such error has been reported by the ISCCP team so far. Another explanation may be that other climatic parameters are acting on cloudiness in addition to atmospheric ionization. A clear decreasing

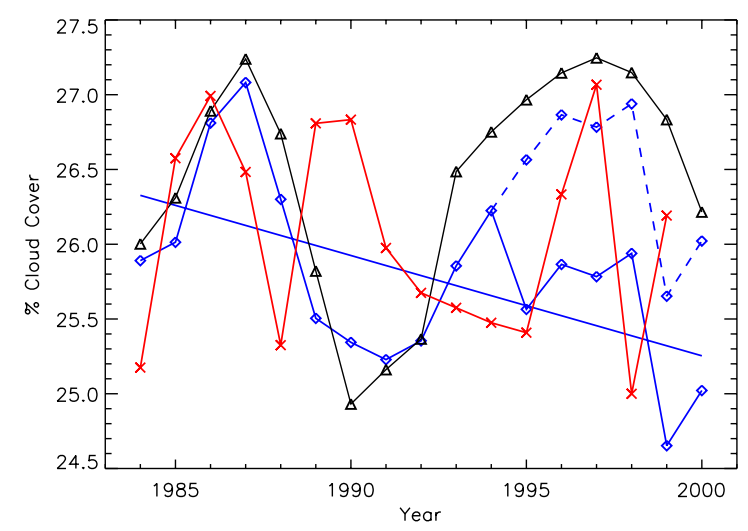

Fig. 2. Global annual mean low cloud cover derived from ISCCP data for the period July 1983-June 2000 (blue diamonds). Also plotted is the mean global GCR-induced ionization level in the atmosphere for the same period (black triangles). The broken line corresponds to the mean low cloud cover with an arbitrary mean level adjustment of $+1 \%$ for the period 1995 onward. The red crosses indicate the el Niño index (normalized to cloud cover scale). 
trend over approximately the past two decades is seen in both the total cloud amount reported by ISCCP (not shown), and the low cloud data (Figs. 2 and 3). A simple linear fit to the yearly low cloud data (Fig. 2) has a slope $-0.065 \% / \mathrm{yr}$. If this trend is subtracted from the low cloud data the correlation coefficient rises from 0.49 to 0.75 , significant at the $99.5 \%$ level.

For a more detailed illustration, in Fig. 3, the filtered monthly mean low cloud cover is plotted against the Climax neutron monitor cosmic ray flux. The detrended low cloud data is also plotted. It is noticeable in the figure how the detrended cloud data has a much better correspondence with cosmic rays. Moreover, it seems that the correspondence between the detrended 12-month filtered low cloud data and the GCR flux is only interrupted during two brief periods around 1993-1994 and 1998-1999.

Next, we look at the correlation between the mean annual low cloud cover for each grid cell and the ionization level from cosmic rays at the mean height of low clouds $(2 \mathrm{~km})$ over the complete 17 years covered by the ISCCP and ionization data (1983-2000). For the correlations reported here, the low cloud data have not been modified in any way. In Fig. 4 we show how the significance of the correlation between these quantities varies across the globe. We note that the region of significant correlation is located over the North and South Atlantic and the south-western Indian Ocean. This geographical distribution is different to that for the data covering the period up to 1994 only (not shown) and it suggests that the location of the region of good correlation may vary.

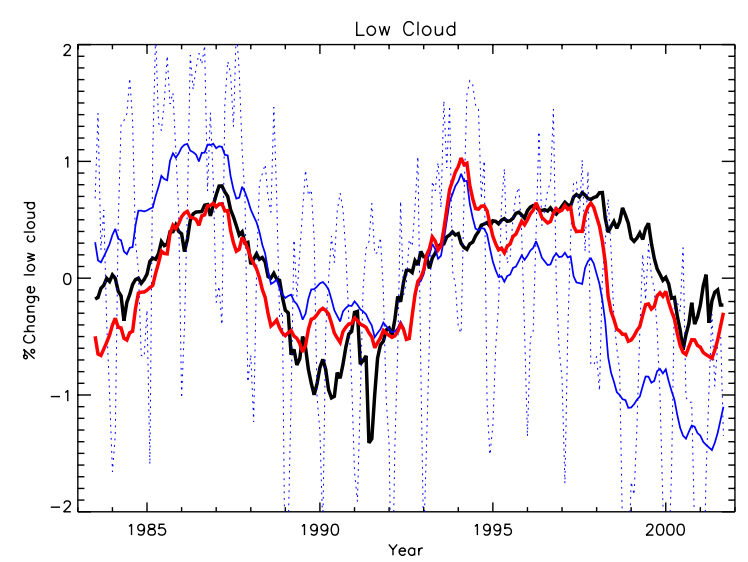

Fig. 3. Global monthly mean low cloud cover derived from ISCCP data for the period July 1983-September 2001 (dotted blue line). Superimposed are the same data filtered with a 12month running mean (blue) and the detrended and filtered (red). The Climax station GCR flux is shown in black. All data are plotted as deviations from the mean for the full period and the cosmic ray flux is arbitrarily scaled.

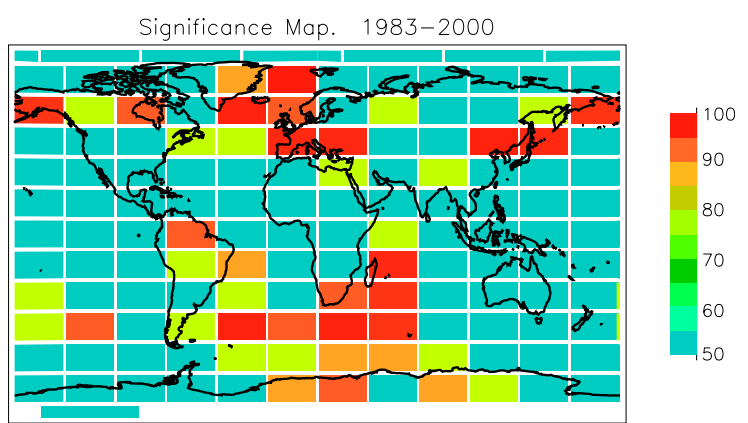

Fig. 4. Global distribution map of the significance of the correlation between low cloud cover and ionization in the atmosphere.

A change in the geographical distribution of the significance of the correlation coefficients, could lead the reader to suspect that any correlation found owes more to a link with the southern oscillation (El Niño) than any cosmic ray induced ionization. To check if this might be the case, we have also computed the correlation coefficients between the mean annual cloud cover and the southern oscillation index (Ropelewski and Jones, 1987). We find no trace of a correlation between these two parameters (there are no boxes with significant correlation either positive or negative even at the $90 \%$ level). However, it is still possible that, although it does not correlate directly with low cloudiness, El Niño is affecting total cloud cover (Farrar, 2000; Marsh and Svensmark, 2003).

With respect to Fig. 4 we note, firstly, that all the significant correlations are positive with the level of low cloudiness increasing as the ionization level increases. There are no grid cells with negative correlations that have a significance equal to or greater than $95 \%$. Secondly, we have repeated (not shown) these correlations using seasonal cloud data rather than annual, and we find that the correlation between low clouds and ionization is neither stronger nor weaker in any particular season.

Thirdly, we note that the grid cells with high degrees of significance are clustered geographically. As mentioned earlier, this is not surprising as field correlations in cloud cover between adjacent cells would be expected. Thus, taken at face value, our results indicate that the spatial correlation pattern is not field significant, and the possibility remains that the correspondence between GCR and low clouds is an artifact of the data.

However, the symmetry in the significance distribution over the northern and southern hemispheres (see next section) points to a physical mechanism behind the correlation. The correlation distribution does become field significant if one looks only at the latitude bands $40-60^{\circ}$ in both hemispheres. Moreover if detrended low cloud data is used, the significances for all cells increase 
and the correlation becomes field significant (although marginally) over the whole earth.

\subsection{Variation of the correlation with latitude and longitude}

Due to the Earth's magnetic field, the flux of galactic cosmic rays is reduced at lower latitudes and reaches its maximum over the magnetic poles. If cosmic rays affect clouds we would expect the effect to be strongest in polar regions and weakest at the equator. This latitudinal effect was first corroborated by Svensmark and Friis-Christensen (1997) for the total cloud cover. However, later work has shown that the correlation of total cloud cover and cosmic rays was not maintained with later (post-1991) satellite data, whereas that for low cloud was (Pallé and Butler, 2000; Sun and Bradley, 2002). Pallé and Butler (2000) found that the correlation for low clouds was stronger in mid-latitude and equatorial belts and weaker in the polar regions. As there were some indications that the low clouds in liquid phase were responsible for the correlation (Pallé, 2001; Palle and Butler, 2000), the reduction in the correlation coefficients poleward was attributed to two factors: (1) the lack of clouds in the liquid phase over the poles and (2) to a reduced reliability of the polar data due to ground ice coverage. The small drop in the degree of correlation at the equator was thought to be due to an increased shielding in the cosmic ray flux (Svensmark and Friis-Christensen, 1997).

To investigate the geographical distribution of the correlation, we have averaged the low cloud and atmospheric ionization in several latitudinal and longitudinal bands, and calculated the correlation coefficients and the slope of the linear fit between these two variables. The latitude bands are $7.5^{\circ}$ wide and longitude bands are $15^{\circ}$ wide, corresponding to the size and location of our cloud grid cells. To test whether our data agrees with the hypothesis that cosmic rays affect low clouds, we are particularly interested in the variation with latitude.

In Fig. 5 we have plotted the correlation coefficient against: (a) geographical latitude, and (c) longitude. Similarly, in Fig. 5(b) and (d) we show the variation of the mean slope of the linear fit. In Fig. 5(a) we see how the correlation drops in all the equatorial and tropical latitude bands, remaining significant only in two latitude belts located between 40 and $60^{\circ}$ North and South. It is worth noticing that it is in these latitude bands that correlations between Forbush decreases of galactic cosmic rays and cloudiness have previously been reported (Todd and Kniveton, 2001; Pudovkin and Veretenenko, 1995). However, both studies suggested that these correlations were associated with high-level cloudiness rather than low clouds. A latitude dependence can also be seen in the slope of the linear fit
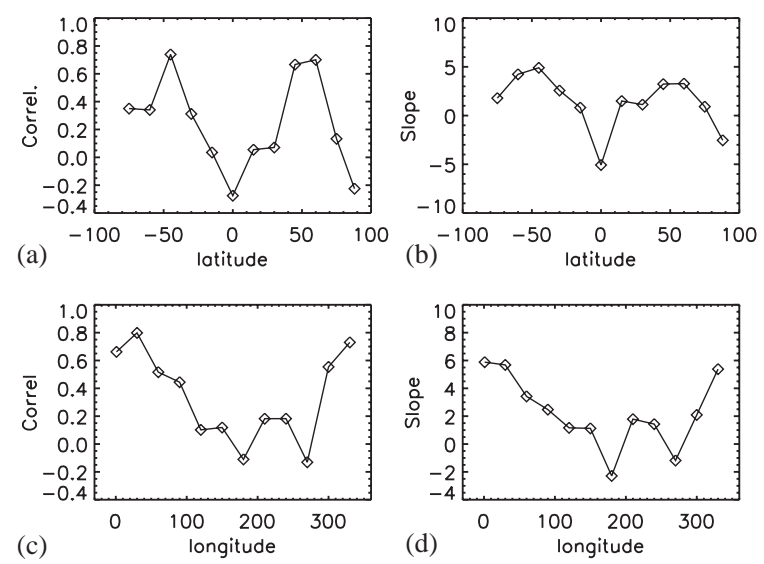

Fig. 5. Dependence of the correlation between low cloud with cosmic ray flux on latitude and longitude. (a) Latitudinal dependence of the correlation coefficient; (b) latitudinal dependence of the slope; (c) longitudinal dependence of the correlation coefficient; (d) longitudinal dependence of the slope.

between low cloud cover and ionization level in Fig. 5 (b).

In Fig. 5(c) we show the variation of the correlation coefficient with longitude. Here, we see the effect referred to earlier, namely a stronger correlation in a particular longitude belt ( -60 to +60 degrees East). The low correlation seen in Fig. 5(c) over the longitude range $100-300^{\circ}$ (east), i.e., the Pacific Ocean suggests that El Niño may have masked any low-cloud cosmic ray correlation in this region during the late 1990s. A similar conclusion has been reached recently by Marsh and Svensmark (2003).

The correlation coefficients over land and ocean have also been calculated separately (not shown); but we have found no significant differences in behavior from the global average. When changing from geographic to geomagnetic latitude, the results are also very similar (not shown).

The above results used data derived directly from the ISCCP without any adjustment for possible changes in calibration after 1994 or the existence of a trend. To examine the possible results of such a trend we have repeated the above computations with detrended cloud data. In general, there is significant improvement in the correlation over the whole Earth. However, the geographical distribution of the correlation remains similar.

The dependence of the correlation on latitude suggests that whichever mechanism might be acting to couple the low cloudiness with the solar signal (or GCR) it operates only in certain latitude bands. This could be taken to indicate that the latitudinal variation is controlled by a combination of at least three factors including: (1) the requirement that the clouds were in a liquid state, (2) the known latitudinal variation in cosmic ray flux, and (3) 
an electroscavenging process operating on liquid clouds (Tinsley and Yu, 2003), dependent on current density changes in the global electric circuit, which have a different latitudinal variation.

\subsection{Possible physical mechanisms for the low cloud- ionization connection}

As evidence for a cloud - cosmic ray connection has emerged, interest has risen in the various physical mechanisms whereby ionization by cosmic rays could influence cloud formation. In parallel with the analysis of observational data by Svensmark and Friis-Christensen (1997), Marsh and Svensmark (2000) and Pallé and Butler (2000), others, including Tinsley (1996), Yu (2002) and Bazilevskaya et al. (2000), have developed the physical understanding of how ionization by cosmic rays may influence the formation of clouds. Two processes that have recently received attention by Tinsley and $\mathrm{Yu}$ (2003) are the IMN process and the electroscavenging process.

IMN involves the promotion of the formation of condensation nuclei $(\mathrm{CN})$ via the intermediary action of sulphuric acid and ionized particles in the atmosphere. The rate at which $\mathrm{CN}$ form is dependent on both the $\mathrm{H}_{2} \mathrm{SO}_{4}$ concentration and the ionization rate by cosmic rays $\mathrm{Yu}$ and Turco (2000), both of which are dependent on ambient conditions and altitude. Yu (2002) has computed the efficiency of the formation of $\mathrm{CN}$ with radii greater than $3 \mathrm{~nm}$ as a function of altitude and has shown that the peak efficiency occurs between 3 and $4 \mathrm{~km}$ altitude, that is near to the altitude of low clouds. Also, whereas these computations predict a positive correlation between cosmic ray ionization and low clouds they predict a negative correlation for high clouds. Though we have not found the negative correlation for ISCCP high cloud data, the IMN theory is apparently able to explain the positive correlation for low clouds.

The second process, considered by Tinsley and $\mathrm{Yu}$ (2003), namely electroscavenging, depends on the action of the global electrical circuit (see review by Rycroft et al. (2000)). The transport of charge by rapidly rising convective currents in the tropics and over continental land masses leads to a $\sim 200 \mathrm{kV}$ positive charge of the ionosphere compared to Earth. This large voltage difference, in turn, necessitates a return current which must pass through the regions of the atmosphere where clouds are formed. As cosmic rays are the principal agent of ionization in the atmosphere above $1 \mathrm{~km}$ altitude, any modulation of the GCR flux due to solar activity is likely to affect the transport of charge to complete the global electrical circuit. Tinsley and $\mathrm{Yu}$ (2003) discuss how the build up of electrostatic charge at the tops and bottoms of clouds could affect the scavenging of ice forming nuclei (IFN) and cloud condensation nuclei $(\mathrm{CCN})$ by droplets, and how this can lead to greater rates of precipitation and a reduction in cloud cover. They find that the electroscavenging process is likely to be more important over oceanic rather than continental regions and that it leads to a positive correlation between clouds and cosmic rays at higher latitudes and a negative correlation at low latitudes. Thus the electroscavenging process can explain several of the most striking features of Fig. 5, namely: (1) the peak in significant positive correlations at latitudes around 50 degrees North and South (Fig. 5a); (2) the tendency for a less significant but nonetheless evident trend to negative correlation coefficients at low latitudes (Fig. 5a); and (3) the location of the peak in correlation over one of the principal oceans, namely over the North and South Atlantic (Fig. 5c).

Although these aspects of our results are consistent with the predictions of the IMN and electroscavenging processes, it is too early to say that they provide real confirmation, particularly in view of the lack of field significance. Other mechanisms which rely on coupling between the upper and lower atmosphere as proposed by Kristjansson et al. (2002) and Haigh (1996) in which solar irradiance changes are the drivers of circulation changes and cloud cover variations remain a possibility.

\section{The amplitudes of low cloud and ionization level over the solar cycle}

If there is a well-defined correlation between the ionization level produced by cosmic rays and the low cloud cover, as has been suggested, then it might be expected that the geographical variation in the amplitude of the ionization level would be similar to that of the amplitude of the low cloud cover. For such a comparison, it is evidently advantageous to use the geographically dependent modeled ionization rather than GCR fluxes from a few isolated stations. We plot the geographical distribution of low cloud amplitude and ionization amplitude over the period covered by the satellite data (1983-2001) in Fig. 6.

In the upper panels of Fig. 6, we show the geographical distribution of: the percentage amplitude change of the ionization level at $2 \mathrm{~km}$ altitude over the solar cyle (top left), and the mean annual low cloud amount 1983-2001 (top right). The ionization amplitude is clearly dependent on the geomagnetic latitude, as expected. It is also evident that mean low cloud levels are more strongly influenced by proximity to the large oceans of the world, than ionization amplitude.

Over the period July 1983 to September 2001 there are two maxima in lower atmosphere ionization rates and one minimum, corresponding to sunspot minima and sunspot maxima respectively. We have averaged the low 

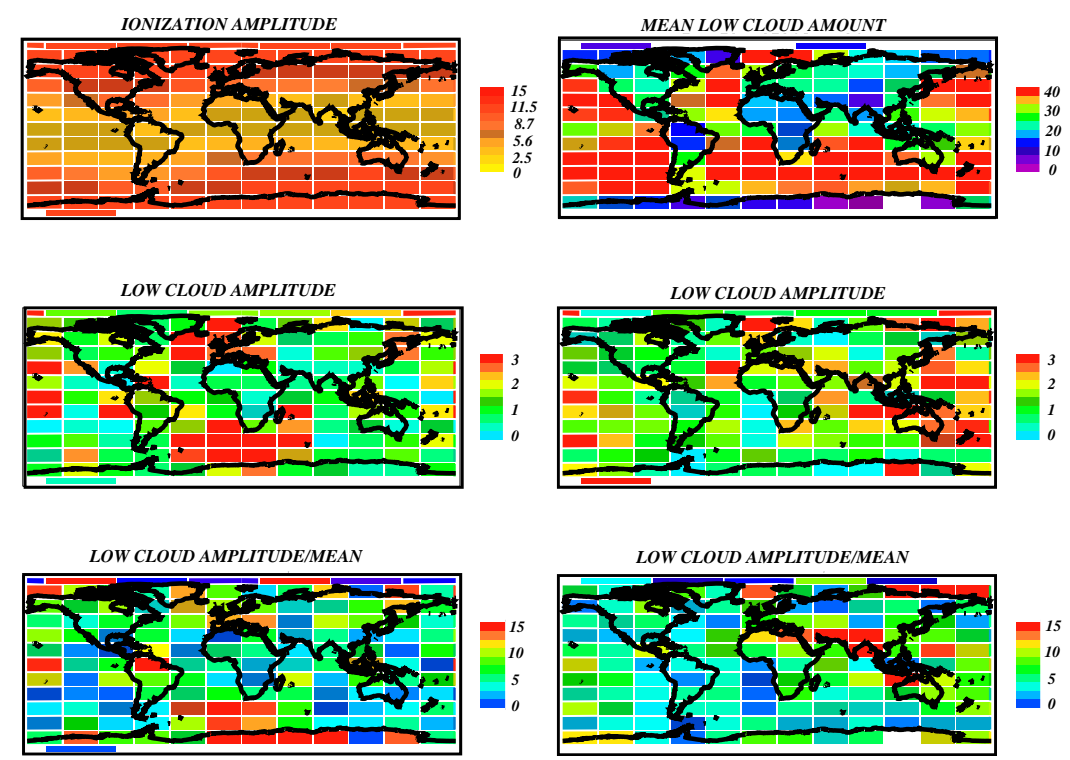

Fig. 6. Top-left, the percentage change in ionization level at $2 \mathrm{~km}$ altitude; Top-right, the ISCCP D2 mean low cloud cover amount for the period July 1983-June 2000; Middle-left, the absolute percentage change in low cloud amplitude from the first maximum to the minimum in atmospheric ionization; Middle-right, the absolute percentage change in low cloud amplitude from minimum to the second maximum in ionization; Bottom left, the percentage change in low cloud amplitude divided by the mean low cloud amount, from the first maximum to the minimum in atmospheric ionization; Bottom-right, as for bottom left but from minimum to the second maximum in ionization.

cloud cover and ionization level for the 3 years around each maximum and minimum and differenced them to form the amplitudes of variation. In Fig. 6, middle left panel, we show the amplitude of low cloud from the first maximum (1985-1988) to the minimum (1991-1993) and, in the middle right panel, from the minimum to the second maximum (1996-1999). In the two lower panels, the relative changes (absolute change over the mean cloud amount) in low cloud amount for the same time periods are shown.

Neither map of cloud amplitude shows any real correspondence to the map of ionization change; the geographical variations seen in the lower four panels of Fig. 6 owe their appearance more to the variation of the mean annual low cloud cover (shown in the top right panel) than to the ionization amplitude. The lack of correspondence between the ionization amplitude map and the low cloud amplitude maps mitigates to some extent against the conclusion that cosmic ray induced ionization is an overriding factor in low cloud variation. Additional factors, such as the geographical variation in the global electrical circuit or concentration of $\mathrm{H}_{2} \mathrm{SO}_{4}$, could be more important in controlling the latitude dependence than cosmic rays (Tinsley, 2003, priv. comm.).

We note that the areas where the correlations were found to be statistically significant in Section 4 correspond quite well to those having the maximum amplitude in low cloud cover during the solar cycle. However, this might have been expected as when the amplitude of variation of cloud cover is small any correlation present would be more easily masked by observational scatter and the significance of the correlation correspondingly reduced. Therefore, in the presence of observational scatter, significant correlations would tend to occur preferentially in regions with a larger amplitude of variation.

$\mathrm{Yu}$ (2002) finds that a $20 \%$ change in ionization produces an approximate $10 \%$ change in IMN at $2 \mathrm{~km}$. In this case, the relative changes in low cloud amount, in areas where the GCR-low cloud correlation is strong, would be expected to be not larger than about half of the relative change in ionization. In Fig. 6, lower panels, we note that some boxes where a significant correlation occurs in Fig. 4, have a percentage change in low cloud of the same order as the percentage change in ionization. This is larger than would be expected from Yu's results. However, this discrepancy is most conspicuous in Antarctica where the low cloud cover is in any case very low and more difficult to measure. The low cloud trend shown in Fig. 2 could probably also explain why the amplitude changes for 1986/8-1991/3 are larger than for the 1991/3-1996/9 period if a correlation with GCR is present. 


\section{Calculating the effects on the Earth's temperature}

In previous sections, it has been shown how the evidence for a connection between GCR and low clouds is inconclusive; though some aspects of our study are encouraging, others are basically negative. Despite these uncertainties, because of the potential impact on the Earth's radiation budget, we have explored the consequences for climate change, should the correlation between low cloud and ionization be confirmed.

Estimates of the possible effect of a link between cosmic rays and clouds have been made previously (Pallé and Butler, 2000; Svensmark, 1998), without taking into account details of the geographical distribution of the low clouds, the strength of the correlation or dependence of the slope of the linear fit on location. We have tried in the present work to repeat these calculations taking what knowledge we have of these parameters into account.

For each of the grid boxes where the correlation (positive or negative) between annual low cloud and ionization at $2 \mathrm{~km}$ was significant at the $95 \%$ level or higher, we have determined a linear fit between ionization and low cloud cover. With the linear fit for the 1983-2000 period established, we have used the mean annual ionization levels calculated by the LUIN code for the period 1868-2000 (see Fig. 7) to predict the annual mean low cloud cover since 1868 for each grid cell in which a significant correlation has been found.

Ockert-Bell and Hartmann (1992) calculated the albedo, outgoing long-wave radiation (OLR) and net radiation $R_{\text {net }}$ at the top of the atmosphere as measured by the Earth Radiation Budget Experiment (ERBE). These radiative properties were determined for each of

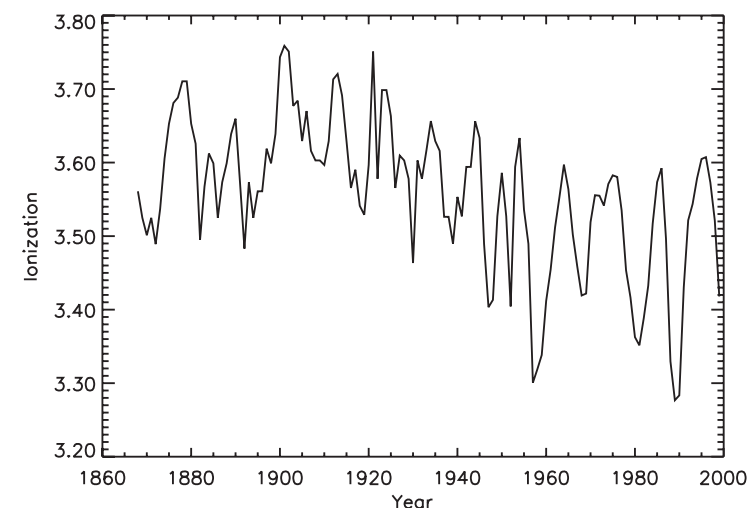

Fig. 7. Global mean annual atmospheric ionization induced by galactic cosmic rays for the period 1868-2000. Units are ionization production rate $\mathrm{cm}^{-3} \mathrm{~s}^{-1}$. Note the decreasing trend in the cosmic ray-induced ionization (i.e., a decrease in the galactic cosmic ray flux reaching the earth's atmosphere) between 1900 and 1950. However, there is no clear systematic trend since the 1960s. the ISCCP cloud types over six characteristic climatic zones of the Earth. Based on the latitude, longitude, land fraction and mean cloud type, we have assigned to each of the grid cells the most appropriate of the six zones. Using their regression values we then calculated the changes in albedo, OLR and $R_{\text {net }}$ due to a change in low cloud coverage during the last $130+$ years for each grid cell. In areas where the correlation was not significant at the $95 \%$ level it has been assumed that no change in low cloud cover occurred, and thus no change in the cloud radiative forcing. There are no areas with a significant but negative correlation.

To calculate a global temperature effect we have added the different radiative effects of all of the 144 cells of our grid, weighted by the total area covered by each grid cell,

$\Delta R_{\mathrm{net}}^{G}(t)=\frac{1}{\sum_{i=1}^{144} A_{i}} \sum_{i=1}^{144} \Delta R_{\mathrm{net}}^{i}(t) A_{i}$,

where $\Delta R_{\text {net }}^{i}$ and $A_{i}$ are the change in $R_{\text {net }}$ and the area of the $i$ th grid cell, respectively, and $\Delta R_{\text {net }}^{G}$ is the global change in $R_{\text {net }}$ in $\mathrm{Wm}^{-2}$.

For consistency with our previous works (Pallé and Butler, 2000) we have used a climate sensitivity of $0.53{ }^{\circ} \mathrm{CW}^{-1} \mathrm{~m}^{2}$, as used by Soon et al. (1996), which is well within the accepted range of $0.3-1^{\circ} \mathrm{CW}^{-1} \mathrm{~m}^{2}$ (Lean and Rind, 1998). Some authors have suggested that the values given in Ockert-Bell and Hartmann (1992) may overestimate the radiative impact of low clouds. However, by choosing a low climate forcing factor in our computations, we should compensate for any overestimate inherent in Ockert-Bell and Hartmann's values. From the above computations we estimate that the effect of a low cloud-ionization connection would be around $0.2^{\circ} \mathrm{C}$ warming during the 20th century; a slightly lower value than the previous estimate of $0.27^{\circ} \mathrm{C}$ (Pallé and Butler, 2000) using a much simpler approximation based on the data from 1983 to 1994 only.

Any cloud-induced change in radiative forcing for the Earth, such as that computed above, must be added to the direct radiative forcing which derives from changes in solar irradiance. In Fig. 8 (left panels) we show for comparison the predicted low cloud induced change in global temperature together with the irradiance-induced change using the results of Lean and Rind (1998) and Lockwood and Stamper (1999). We note that the low cloud-induced radiative forcing, when summed globally, is rather less than that produced by irradiance change. This conclusion is based on the un-detrended 17 year cloud dataset and differs from that based on the 11-year dataset reported previously (Pallé and Butler, 2000).

In the right-hand side panels of Fig. 8, we show the sum of the two solar activity related forcings, namely the direct effect of irradiance change and the suggested lowcloud cosmic ray connection, together with the observed 

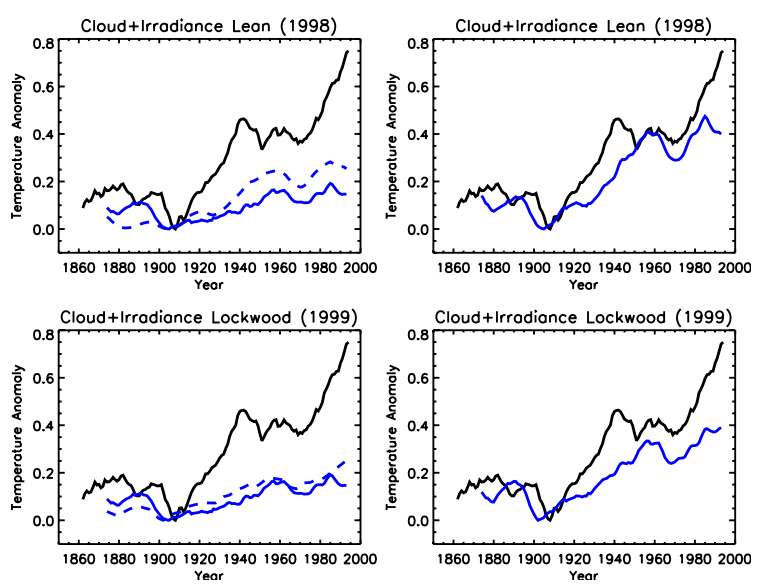

Fig. 8. Measured and predicted temperature changes during the last 150 years. The thick black line in all panels is the measured global temperature anomaly by Jones et al. (2001). Top left: the 11-y filtered predicted temperature anomalies from low cloud variation (solid blue line) and the 11-y filtered predicted temperature anomalies series by Lean and Rind (1998) from changes in solar irradiance (dotted line). Top right: the contributions of the solar direct (irradiance) plus indirect (low cloud) forcing plotted in the top left panel are combined and compared to the instrumental record. Bottom panels: Same as for top panels respectively but using the irradiance estimates by Lockwood and Stamper (1999). The zero level of the reconstructed temperatures has been arbitrarily scaled to coincide to that of the measured temperatures.

global temperature anomaly of Jones et al. (2001). There is relatively good agreement between the observed anomaly and the combined curves for the period 1870-1910, but increasing divergence from 1910 onward. By the 1990s, the difference is of the order one third to one half of the global warming since the late 19th century.

Thus it appears that, provided further satellite cloud data confirms the cosmic ray flux low cloud seeding hypothesis, and no other factors were involved over the past 150 years (e.g., variability of other cloud layers) then there is a potential for solar activity induced changes in cloudiness and irradiance to account for a significant part of the global warming experienced during the 20th century, with the possible exception of the last two decades.

However, lest the previous sentence is taken out of context, we should point out that there is clear evidence that total cloud cover has changed over the past 150 years (Pallé and Butler, 2001, 2002). If this were confirmed then the predictions above are incomplete as changes in cloud at other levels will also alter the energy balance with a contribution that could be either positive or negative.

Furthermore there is a trend in ISCCP total and low cloud cover during the period 1983-2001, which in principle, will act to accentuate the forcing described in this section. Neither have we considered in this paper the climatic impact of changes in greenhouse gasses concentration in the atmosphere, the role of volcanic activity, the role of atmospheric aerosols or the internal variability of the climate system. Thus, the climate forcing described in this section is but one of the several parameters contributing to climate change. Not until we have reliable long-term measurements of cloud at all heights, can we draw any really firm conclusions as to the long-term changes in cloud radiative forcing.

\section{Conclusions}

- The correlation between annual mean low cloud and the ionization level at $2 \mathrm{~km}$ altitude exceeds the $99 \%$ significance level over mid-latitude oceans and globally over the period 1983-1994. However, globally, it drops to non-significant values if the full available cloud dataset (1983-2001) is taken into account, although some data adjustment such as detrending can restore the correlation significance to $99.5 \%$ or greater. Nonetheless, the correlation is significant over several large areas of the earth.

- The percentage of grid cells over the globe with a positive correlation coefficient between mean annual low cloud cover and ionization level is higher than would occur by chance if each cell were to be independent. However, on a global basis, field correlations in the cloud data could account for the degree of correlation seen thereby rendering the correlations not field significant.

- There are geographical variations in the degree of correlation between cosmic ray induced ionization at $2 \mathrm{~km}$ altitude and low level clouds. The correlation is stronger and field-significant in mid-latitudes $\left(40-60^{\circ}\right.$ $N \& S)$ than in high latitudes and equatorial regions. Also, there is some evidence for a possibly unstable variation with longitude; we find the regions with strongest correlation over the period 1983-2000 to be located over the North and South Atlantic, whereas data from the more restricted period 1983-1993 show a stronger correlation over the Pacific. The variation of the degree of correlation with latitude is consistent with the predicted behavior of the electroscavenging process described by Tinsley and Yu (2003).

- Our use of modeled GCR-induced ionization allows us to calculate the geographical distribution of the variations in this quantity from maxima to minima of solar activity. If the correlation between the low cloud cover and ionization level were real, it might be expected that the amplitude of the variation in the low cloud cover over the sunspot cycle would have a similar geographical variation to that of the amplitude 
of the ionization level. However, there is no apparent correspondence.

- Assuming that the correlation between the low cloud cover and the ionization level is real, and using the regression coefficients determined from the 1983-2001 data, we can estimate global temperature anomalies from predicted changes in cloud cover over the past 150 years. When combined with the additional forcing by the solar irradiance change, we get predicted global temperature anomalies with some similarity to those from observations, however an upward trend in temperature still remains. The extent of this residual warming is crucially dependent on the climate sensitivity assumed - a higher value for this parameter would reduce the discrepancy. On the other hand, the residual could be due to greenhouse gas warming, with contributions from changes in cloud cover, solar irradiance and greenhouse gases all contributing significantly to the global temperature changes over the past 150 years.

\section{Acknowledgements}

The authors would like to thank the several anonymous referees for their comments and ideas which led to the improvement of this manuscript. Also, we would like to thank Dr Brian Tinsley for comments. The cloud D2 datasets were obtained from the NASA Langley Research Center Atmospheric Sciences Data Center. Research at Big Bear Solar Observatory is supported in part by a grant from NASA (NAG5-11007). Research at Armagh Observatory is grant-aided by the Department of Culture, Arts and Leisure for Northern Ireland.

\section{References}

Alsmiller, F.S., 1965. A general category of soluble nucleon-meson cascade equations. ORNL, 3746.

Bazilevskaya, G.A., Krainev, M.B., Makhmutov, V.S., 2000. Effects of cosmic rays on the earth's environment. Journal of Atmospheric and Solar-Terrestrial Physics 62, 1577-1586.

Carslaw, K.S., Harrison, R.G., Kirkby, J., 2002. Cosmic rays, clouds and climate. Science 298, 1732-1736.

Elliott, J.P., 1955. Proceedings of the Royal Astrogical Society 228A, 424.

Farrar, P.D., 2000. Are cosmic rays influencing oceanic cloud coverage or is it only El Niño? Climatic Change 47, 7-15.

Haigh, J.D., 1996. The impact of solar variability on climate. Science 272, 981-984.

Jones, P.D., Osborn, T.J., Briffa, K.R., Folland, C.K., Horton, E.B., Alexander, L.V., Parker, D.E., Rayner, N.A., 2001. Adjusting for sampling density in grid box land and ocean surface temperature time series. Journal of Geophysical Research 106, 3371-3380.
Kernthaler, S.C., Toumi, R., Haigh, J.D., 1999. Some doubts concerning a link between cosmic ray fluxes and global cloudiness. Geophysics Research Letters 26, 863-865.

Kristjansson, J.E., Staple, A., Kristiansen, J., Kaas, E., 2002. A new look at possible connections between solar activity, clouds and climate. Geophysics Research Letters 29 (23), 2107-2111.

Lean, J., Rind, D., 1998. Climate forcing by changing solar radiation. Journal of Climate 11, 3069-3094.

Lockwood, M., Stamper, R., 1999. Long-term drift of the coronal source magnetic flux and the total solar irradiance. Geophysics Research Letters 26, 2461-2464.

Lowder, W.M., Raft, P.D., Beck, H.L., Warman, E.A. (Eds.), 1971. Experimental determination of cosmic-ray charged particle intensity profiles in the atmosphere. Proceedings of the National Symposium on Natural and Man-made Radiation in Space. National Aeronautics and Space Administration Report, NASA TM X-2440, pp. 908-913.

Marsden, D., Lingenfelter, R.E., 2003. Solar activity and cloud opacity variations: a modulated cosmic ray ionization model. Journal of Atmospheric Science 60, 626-636.

Marsh, N., Svensmark, H., 2000. Low cloud properties influenced by cosmic rays. Physics Review Letters 85, 5004-5007.

Marsh, N., Svensmark, H., 2003. Galactic cosmic ray and El Niño-Southern oscillation trends in international satellite cloud climatology project D2 low-cloud properties. Journal of Geophysical Research 108, 4195.

O'Brien, K., 1979. Secular variations in the production of cosmogenic isotopes in the Earth's atmosphere. Journal of Geophysical Research 84, 423.

Ockert-Bell, M.E., Hartmann, D.L., 1992. The effect of cloud type on Earth's energy balance: results for selected regions. Journal of Climate 5, 1157-1171.

Pallé, E., 2001. Solar activity, cloud cover and climate change. Ph.D. Thesis, Queen's University of Belfast.

Pallé, E., Butler, C.J., 2000. The influence of cosmic rays on terrestrial clouds and global warming. Astronomy and Geophysics 41, 18-22.

Pallé, E., Butler, C.J., 2001. Sunshine records from Ireland, cloud factors and possible links to solar activity and cosmic rays. International Journal of Climatology 21, 709-729.

Pallé, E., Butler, C.J., 2002. The proposed connection between clouds and cosmic rays: cloud behavior during the past 50-120 years. Journal of Atmospheric and Solar-Terrestrial Physics 64 (3), 327-337.

Passow, C., 1962. Phenomenologische Theorie zur Berechnung einer Kaskade aus schweren Teilchen (Nukleonenkaskade) in der Materie (Phenomenological theory for the calculation of a cascade of heavy particles (nucleonic cascade) in matter). Notiz, A 285, Deut. Elektron. Synchrotron, Hamburg.

Pudovkin, M.I., Veretenenko, S.V., 1995. Cloudiness decreases associated with Forbush decreases of galactic cosmic rays. Journal of Atmospheric and Solar-Terrestrial Physics 57, 1349-1355.

Ropelewski, C.F., Jones, P.D., 1987. An extension of the Tahiti-Darwin Southern Oscillation Index. Monthly Weather Review 115, 2161-2165.

Rossow, W.B., Walker, A.W., Beuschel, D.E., Roiter, M.D., 1996. International Satellite Cloud Climatology Project 
(ISCCP): documentation of New Cloud Datasets. WMO/ TD-No. 737, World Meteorological Organization, Geneva, $115 \mathrm{pp}$.

Rycroft, M.J., Israelsson, S., Price, C., 2000. The global atmospheric circuit, solar activity and climate change. Journal of Atmospheric Solar-Terrestrial Physics 62, 1563-1576.

Shindell, D., Rind, D., Balachandran, N., Lean, J., Lonergan, P., 1999. Solar cycle variability, ozone and climate. Science 284, 305-308.

Soon, W.H., Posmentier, E.S., Baliunas, S.L., 1996. Inference of Solar Irradiance variability from terrestrial temperature changes, 1880-1993: an astrophysical application of the Sun-Climate connection. Astrophysical Journal 472, 891-902.

Sun, B., Bradley, R.S., 2002. Solar influences on cosmic rays and cloud formation: a reassessment. Journal of Geophysical Research 107 (D14) doi:10.1029/2001JD000560.

Svensmark, H., 1998. Influence of cosmic rays on Earth's climate. Physics Review Letters 81, 5027-5030.

Svensmark, H., Friis-Christensen, E., 1997. Variations of Cosmic Ray Flux and global cloud coverage. A missing link in solar-climate relationships. Journal of Atmospheric and Solar-Terrestrial Physics 59, 1225-1232.

Tinsley, B.A., 1996. Correlations of atmospheric dynamics with solar wind-induced changes of air-earth current density into cloud tops. Journal of Geophysical Research 101, 29701-29714.

Tinsley, B.A., Yu, F., 2003. Atmospheric ionization and clouds as links between solar activity and climate. In: Frolich, C.,
Hudson, H., Kuhn, J., McCormack, J., North, J., Pap, J., Sprigg, W., Wu, S.T. (Eds.), AGU monograph: solar variability and its effects on the earth's atmospheric and climate system, in press.

Tinsley, B.A., Rohrbaugh, R.P., Hei, M., Beard, K.V., 2000. Effects of image charges on the scavenging of aerosol particles by cloud droplets, and on droplet charging and possible ice nucleation processes. Journal of Atmospheric Sciences 57, 2118-2134.

Todd, M.C., Kniveton, D.R., 2001. Changes in cloud cover associated with Forbush decreases of galactic cosmic rays. Journal of Geophysical Research 106 (D23), 32031-32041.

Turco, R.P., Zhao, J., Yu, F., 1998. A new source of tropospheric aerosols: ion-ion recombination. Geophysical Research Letters 25, 635-638.

Udelhofen, P.M., Cess, R.D., 2001. Cloud cover variations over the United States: an influence of cosmic rays or solar variability? Geophysics Research Letters 28, 2617-2620.

White, W.B., Lean, J., Cayan, D.R., Dettinger, M.D., 1997. Response of global upper ocean temperature to changing solar irradiance. Journal of Geophysical Research 102, 3255-3266.

Williams, M.M.R., 1966. Nucleonik 9, 305.

$\mathrm{Yu}, \mathrm{F}$., 2002. Altitude variations of cosmic ray induced production of aerosols: implications for global cloudiness and climate. Journal of Geophysical Research 107 (A7) doi:10.1029/2001JA0D0248.

Yu, F., Turco, R.P., 2000. Ultrafine aerosol formation via ionmediated nucleation. Geophysics Research Letters 27, 883-886. 